\title{
Glaucoma related retinal oximetry: a technology update
}

This article was published in the following Dove Press journal:

Clinical Ophthalmology

\author{
Zhu Li Yap ${ }^{1,2}$ \\ Sushma Verma \\ Yi Fang Lee ${ }^{1,2}$ \\ Charles Ong ${ }^{1,2}$ \\ Aditi Mohla ${ }^{1,2}$ \\ Shamira A Perera ${ }^{1,2}$ \\ 'Singapore National Eye Center, \\ ${ }^{2}$ Singapore Eye Research Institute, \\ Singapore
}

Correspondence: Shamira A Perera Singapore National Eye Center, II Third Hospital Avenue, Singapore 16875 I

Tel +6563224584

Fax +6563231903

Email shamira.perera@snec.com.sg

\begin{abstract}
There are two long-standing theories about the pathogenesis of glaucoma - barotrauma and the effect of vascular hypoxia. Currently, it is still unknown whether diminished blood flow is the cause or result of glaucomatous atrophy of ganglion cells and the optic nerve. Though many other imaging techniques used to directly assess ocular blood flow have been well studied, they are limited by their inability to directly assess metabolism in the ocular tissues or measure the oxygen carrying capacity in the vessels. Retinal oximetry is a relatively novel, noninvasive imaging technique that reliably measures oxygen saturation levels in the retinal vessels, offering surrogate markers for the metabolic demands of the eye. The clinical significance of these measurements has not been well established. Thus, this review gives an overview of ocular imaging and current retinal oximetry techniques, while contextualizing the important oximetry studies that have investigated the vascular theory behind glaucoma.
\end{abstract}

Keywords: retinal oximetry, glaucoma, Oxymap, retinal vasculature, glaucoma imaging

\section{Introduction}

Retinal oximetry is a relatively novel, noninvasive imaging technique that reliably measures oxygen saturation levels in retinal vessels, acting as a surrogate marker for the metabolic demands of the eye.

It is widely accepted that retinal blood flow or oxygenation is altered in vasculopathies such as retinal vessel occlusions and diabetic retinopathy. There is also mounting evidence implicating vascular compromise in glaucoma. ${ }^{1-3}$ Measurements of retinal oxygenation in such conditions help improve our understanding of pathophysiology and may guide us in making diagnostic and therapeutic decisions.

Glaucoma is an optic neuropathy associated with retinal ganglion cell death and visual field loss. ${ }^{1,4}$ Two long-standing theories explain different, but inter-related, mechanisms involved in the pathogenesis of the disease. The mechanical theory of barotrauma to the nerve and subsequent cell death has been thought to be the predominant cause in high-pressure glaucomas such as primary open-angle glaucoma (POAG) or primary angle closure glaucoma (PACG). ${ }^{5}$ The vascular theory, in which there is hypoperfusion, ischemia, hypoxia and ensuing tissue damage, is thought to play a major role in normal tension glaucoma (NTG). ${ }^{6}$

Many studies have shown dysfunctional regulation of ocular blood flow to be associated with increased incidence, prevalence and progression of glaucoma. ${ }^{6-8}$ There is growing evidence that blood flow in glaucomatous eyes is reduced or that its regulation is impaired compared with normal, non-glaucomatous eyes. ${ }^{1,8}$ Corroborating this hypothesis, blood flow deficiencies have been reported in both the choroidal and retrobulbar circulations in glaucoma patients. ${ }^{9-15}$ Plange et al showed that in asymmetric 
glaucoma, the blood velocity in the central retinal artery was reduced in the more affected eye, ${ }^{16}$ while Sato et al found that in asymmetric NTG eyes, the neuroretinal rim blood flow was reduced in areas corresponding to scotoma. ${ }^{17}$

In addition, systemic and localized vascular abnormalities have been associated with POAG, ${ }^{18-21}$ while reduced ocular perfusion pressure has been shown to have links with both the prevalence and incidence of glaucoma. ${ }^{22-24}$ There has also been speculation that vascular autoregulation is impaired in glaucoma, which results in impaired blood flow and energy metabolism. ${ }^{6,25}$ The evidence from all these studies strongly implicates vascular factors in the pathogenesis of glaucoma.

The role of hypoxia is further supported by the increased staining of hypoxia-induced factor- $1 \alpha$ in the retina and optic nerve of patients with glaucoma compared with healthy individuals. ${ }^{26,27}$ Animal studies have demonstrated that the oxygen tension at the optic nerve decreases when the intraocular pressure (IOP) rises to high levels and increases when the IOP is lowered again. ${ }^{28}$

Currently, it is still unknown whether diminished blood flow is the cause or result of glaucomatous atrophy of ganglion cells and the optic nerve.

\section{Ocular imaging and retinal oximetry techniques}

Many imaging techniques used to directly assess ocular blood flow have been well studied, such as fundal fluorescein angiography, color Doppler imaging, confocal scanning laser ophthalmoscopic angiography, Canon laser blood flowmetry and scanning laser Doppler flowmetry. ${ }^{29}$ The limitation of these techniques is the inability to directly assess metabolism in the ocular tissues or measure oxygen-carrying capacity in the vessels.

Retinal oxygenation can be measured with either invasive or noninvasive methods. Retinal oximetry is a new, emerging noninvasive technique that can reliably provide us with important information of retinal metabolism by measuring oxygen saturation levels in retinal vessels. The two main systems currently in use are the Oxymap T1 Retinal Oximeter (Oxymap, Reykjavik, Iceland; Figure 1) and the Vesselmap system (Imedos Systems UG, Jena, Germany; Figure 2).

While different retinal oximetry systems have different constructs, the basic structure consists of a fundus camera with an attached image splitter. The image splitter contains mirrors that split the beam from the camera into smaller beams based on wavelengths of light, as well as filters that further filter the beam. The apparatus also includes a digital camera to capture the fundus images.

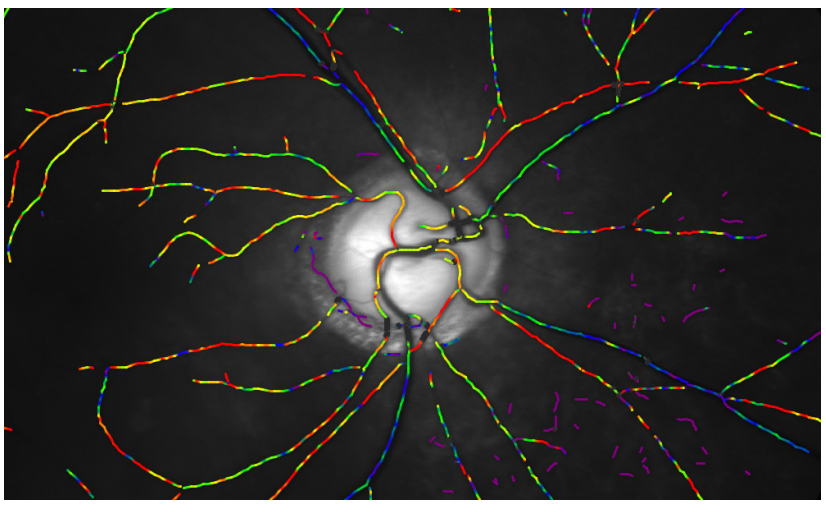

Figure I Image from the Oxymap system showing oxygen saturation in the retinal vessels. ${ }^{30}$

Analysis of the images has been shown to be most effective with comparison of the images at two distinct wavelengths, at $600 \mathrm{~nm}$ (sensitive to oxyhemoglobin) and at about $570 \mathrm{~nm}$ (insensitive to oxyhemoglobin). Computer software detects retinal vessels and uses relative light intensities to calculate relative vessel oxygenation. The OD of retinal vessels from the two acquired images is automatically calculated by software algorithm according to the equation $\mathrm{OD}=\log (\mathrm{I} 0 / \mathrm{I})$, where $\mathrm{I} 0$ is the light reflected by the background to the side of the vessel and I is the light reflected from the vessel. The ratio of OD at $600 \mathrm{~nm}$ and OD at $570 \mathrm{~nm}$ is inversely related to hemoglobin oxygen saturation. ${ }^{2,31,32}$

Many recent studies have been performed using the Oxymap system. The device is designed to capture and measure retinal vessel oxygen saturation in one comprehensive system using a fundus camera add-on and uses spectrophotometric measurements. Although it has been shown to have repeatable results in small studies when imaging the same

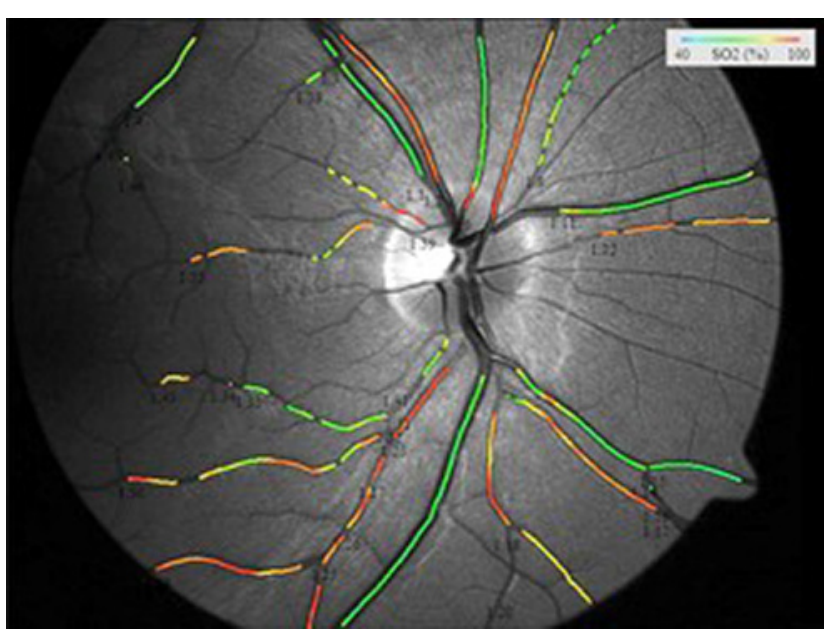

Figure 2 Image from the Vesselmap system showing oxygen saturation in the retinal vessels.

Note: Copyright Imedos Systems UG. ${ }^{55}$ 


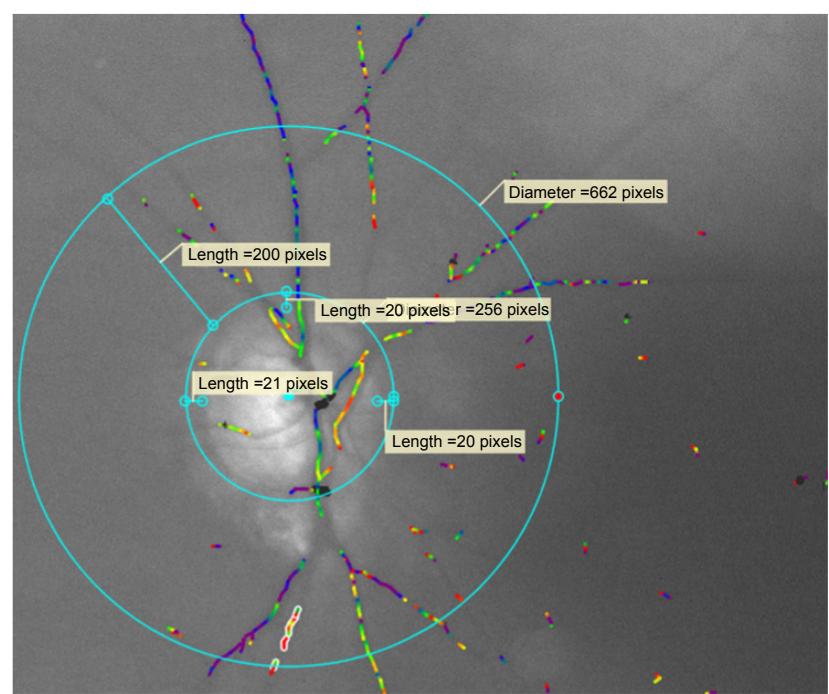

Figure 3 Image from the Oxymap Analyzer showing disrupted vessel detection due to the presence of a cataract. ${ }^{30}$

retinal region, the vessel detection and, hence, the oximetry analysis are often adversely affected in the presence of cataracts (Figure 3 ). As a result, the clinical significance of these measurements has not been well established..$^{33,34}$

\section{Alternative approaches}

A few novel approaches have been utilized in an attempt to capture multiple wavelengths in a snapshot. Ramella-Roman et al used a lenslet array and different filters behind each lenslet. ${ }^{35-37}$ This allows capture of an image at several different wavelengths simultaneously. Testing on samples of hemoglobin and on healthy volunteers was carried out with six wavelengths of light. Harvey et al have developed an instrument that captures information on eight wavelengths simultaneously using spectral demultiplexing. ${ }^{35,38}$ The same group of authors have also used another system to develop and test their oximetry calculations; it records information on different wavelengths sequentially. Yet another approach to multi-/hyperspectral retinal oximetry was taken by Johnson et al who used a method based on hologram technology to separate up to 50 wavelengths in snapshot images. ${ }^{35,39}$ There has been limited use of the system in humans, but validation results from rabbits, using 28 wavelengths, were recently published..$^{35,40}$

Noninvasive oximetry has mainly been used to study blood in the larger retinal vessels. Attempts have been made to measure outside the larger vessels with multi-wavelength oximeters, with those measurements being made of the optic nerve head and the retina. ${ }^{41-43}$ Recently, Desjardins et al published preliminary data using hyperspectral retinal camera which focussed on relative oximetry and total hemoglobin outside of the main vessels. Even though it is a very small study, nonetheless, this could be a step in the direction to obtaining further data which could further elucidate on the topic matter. The main challenge in these studies is to isolate a small signal from the capillaries of interest. ${ }^{44}$

Yet another study by Gonzalez-Hernandez et al looked at optic nerve head color images and used the Laguna ONhE program (Insoft SL, Tenerife, Spain) to estimate the hemoglobin amount in each sector. Within the very limited area of the disc, they found optic nerve head (ONH) hemoglobin correlated well with retinal nerve fibre layer (RNFL) thickness, except in the nasal and temporal sectors. ${ }^{45}$

\section{Retinal oximetry in glaucoma Retinal oxygenation and glaucoma pathophysiology}

Retinal oximetry is a promising tool for its potential in directly assessing oxygen saturation. In particular, we explore how it can contribute to further supporting the vascular theory of glaucoma.

When Michelson and Scibor measured oxygen saturation in healthy persons and in patients with POAG and NTG, they found a correlation between the arteriovenous (AV) difference in oxygen saturation and the rim area in patients with glaucoma, but did not find a correlation between arteriolar oxygen saturation and mean defect/visual field defect or rim area. ${ }^{3}$ The limitation of their study was that they only measured a small segment of the superior temporal retinal arteriole and venule, $\sim 1-3 \mathrm{~mm}$ from the disc margin, which gives limited information on the overall retinal metabolism. Changes in oxygenation at the superior temporal region may not account for the glaucomatous changes in other quadrants. However, they did concur that their data showed a glaucomatous loss of rim tissue indicating a chronic process is coupled with changes in retinal oxygen saturation.

In addition, they also found that arteriolar saturation was slightly lower in NTG as compared to that in healthy volunteers. This may be related to poor ocular blood flow in those patients.

Conversely, a few studies found that in patients with treated POAG, deeper visual field defects were associated with increased oxygen saturation in venules and decreased AV difference in retinal oxygen saturation. ${ }^{1,46}$ The most likely explanation is that glaucomatous atrophy decreases the demand for oxygen, resulting in a higher retinal venous oxygen saturation. The data suggested that oxygen metabolism is affected in the glaucomatous retina, possibly related to tissue atrophy, adding further to the evidence for ischemia/ hypoxia in glaucoma. It should be noted that some of their 
patients had also undergone glaucoma surgery which has been postulated to increase oxygen saturation. ${ }^{47}$

Similarly, Vandewalle et al showed that in patients with treated open-angle glaucoma, severe glaucomatous damage is associated with increased oxygen saturation in the first- and second-degree retinal venules and decreased AV difference in retinal oxygen saturation. Moreover, they found that the AV difference in oxygen saturation decreased as the rim area and the nerve fiber layer decreased. ${ }^{26}$

In their comparison of retinal oxygen saturation in patients with POAG and healthy subjects, Ito et al found a correlation in patients with glaucoma between mean visual field defect and average oxygen saturation only in the inferoand supero-temporal region of the retina. ${ }^{48}$

Furthering this, Olafsdottir et al also found that patients with advanced open-angle glaucoma had higher oxygen saturation in the venules and lower AV differences in oxygen saturation compared with healthy individuals. ${ }^{2}$ They speculated the decreased AV difference in severe glaucoma may be related to lower oxygen consumption secondary to tissue atrophy, which produced lower oxygen requirements. This study also showed that there was no difference in the retinal oxygen saturation between healthy individuals and patients with glaucoma, suggesting that hypoxia is unlikely to be the primary cause or consequence of ischemia.

Delving deeper, Cheng et al compared retinal oximetry and vessel caliber between eyes of asymmetrical glaucoma severity and of normal subjects. ${ }^{49}$ They found that compared to normal controls, arteriolar oxygen saturation was increased in PACG eyes, but not in POAG or NTG eyes. Uniquely, they found there were no significant differences in oxygen saturation in the venules or in $\mathrm{AV}$ difference between the three glaucoma subgroups. Venular diameter was significantly reduced in all glaucoma groups compared to normal controls, but no such change was observed in arteriolar diameter.

Interestingly, when divided according to severity, it was also noted that in PACG eyes with more severe disease, the arteriolar diameter was significantly lower and the arteriolar oxygen saturation and AV difference were significantly higher. This trend was not observed in POAG or NTG eyes with asymmetrical disease. We hypothesize that the oximetry difference observed in PACG eyes may be due to an increased metabolic demand in the disease process compared to openangle glaucoma.

With regard to the vessel caliber, it is possibly due to Poiseuille's formula, in which the volume flow varies to the fourth power of the radius of the tube, provided the pressure gradient remains constant. In short, a small decrease in vessel radius can significantly decrease the volume flow rate, as well as cause increased vascular resistance and lead to alteration of oxygen provision to the retina and optic nerve. Their study was limited by the lack of data about the effect of IOP on pressure gradient and vessel caliber at the onset of the disease; also, they observed patients who had received treatment for glaucoma.

In our more recently published study of retinal oximetry in subjects with glaucomatous hemifield asymmetry, we noted that localized visual field losses were not associated with changes in retinal oximetry, but were associated with narrower retinal arteriolar diameters in the same hemifield of PACG and NTG patients. These same patients also demonstrated marginally higher venular oxygen saturation and a smaller AV difference in the more affected hemifield, but this was not statistically significant. The retinal nerve fiber layer was thinner in the more affected hemifield in these two groups, but was not so marked in the POAG eyes, possibly limiting our ability to find a difference in arteriolar diameter there.$^{30}$ It is possible that the oximetry changes do not localize well to the visual field defects or that the differences between the more and less affected hemifields were not large enough to produce significant results; previous studies only found significant differences in oximetry when they compared healthy controls with patients who had advanced glaucoma. Unfortunately, retinal blood flow was not quantified in our study and this is an important determinant of retinal perfusion and subsequent hemifield loss.

\section{Retinal oxygen saturation and glaucoma drug treatment}

From the literature, it is known that some medications can have an effect on the retinal oxygen saturation by influencing ocular blood flow. In a literature review and meta-analysis, Siesky et al looked at an 8-month treatment with dorzolamidetimolol or timolol alone and found that the decrease in IOP with dual therapy decreased IOP and increased retinal blood flow in the superficial retinal vasculature in both glaucomatous and healthy patients. ${ }^{50}$ While there was no significant effect on oximetry measurements in the retinal arteries and veins, the combination of increased retinal blood flow with consistent oxygen saturation may potentially increase oxygen delivery to the retina. Correspondingly, Traustason et al showed that switching from the combination of dorzolamide-timolol to timolol monotherapy resulted in decreased arteriolar and venular oxygen saturation. ${ }^{51}$ It has also been shown that both brinzolamide and dorzolamide increased oxygen saturation in the superior and inferior retinal veins, although no significant correlation was found in the retinal arteries. ${ }^{52}$ 


\section{Retinal oxygen saturation and glaucoma surgery}

Hardarson et al looked at the effect of glaucoma filtration surgery on retinal oxygen saturation and found a $2 \%$ oxygen increase in oxygen saturation in arterioles $\sim 1$ month after trabeculectomy compared to presurgical values, with no significant change in venular oxygen saturation. They concluded that glaucoma filtration surgery, which lowers IOP, results in almost no change in retinal vessel oxygen saturation. ${ }^{47}$ A more recent study by Nitta et al found that although oxygen saturation in retinal arterioles remained unchanged before and after glaucoma surgery, either trabeculectomy or EXPRESS (Alcon Laboratories, Fort Worth, TX, USA) shunt surgery, the oxygen saturation in the venules increased and concluded that the IOP decrease caused by glaucoma surgery had an effect on the retinal venous oxygen saturation..$^{53}$ Given the complex nature of glaucoma, the studies were limited by a small size and not having well-defined criteria, for example, not controlled for severity, type of glaucoma and randomization to surgery or not.

While the posttreatment retinal venous saturation seems to have remained largely unchanged in most of these studies, the lack of large or consistent changes in either direction may also provide indirect clues, supporting that these changes may be due to the disease rather than the cause. Also, there is likely to be some variation due to the racial differences in cutaneous and fundus pigmentation that has skewed oximetry readings.

\section{Conclusion}

Retinal oximetry is a new noninvasive technology that has potential for use in a clinical setting, as it provides measurements of retinal vessel oxygen saturation in health and disease. It is important to note that significant media opacity and poor contrast as well as light scatter are likely to affect measured oximetry. We have reviewed the most pertinent studies in the context of glaucoma where it has aided in the understanding of the pathophysiology and, to a certain extent, shows promise in guiding diagnostic and therapeutic decisions.

There are numerous other ocular diseases in which retinal oximetry is likely to play a more significant role, in particular, retinal vascular diseases such as diabetic retinopathy, retinal vein and artery occlusions and ocular ischemic syndrome $\mathrm{e}^{35,43,54}$ as well as central nervous system diseases. ${ }^{52}$ Similarly, the Oxymap system has a utility in evaluating eyes after vitrectomy, where authors found both retinal arteriolar and venular oxygen saturations were significantly increased. ${ }^{53}$ However, to be incorporated into our routine clinical work-up, oximetry needs to show value across a variety of clinical situations that will ultimately change management.

\section{Disclosure}

The authors report no conflicts of interest in this work.

\section{References}

1. Olafsdottir OB, Hardarson SH, Gottfredsdottir MS, Harris A, Stefánsson E. Retinal oximetry in primary open-angle glaucoma. Invest Ophthalmol Vis Sci. 2011;52(9):6409-6413.

2. Olafsdottir OB, Vandewalle E, Abegão Pinto L, et al. Retinal oxygen metabolism in healthy subjects and glaucoma patients. Br JOphthalmol. 2014;98(3):329-333.

3. Michelson G, Scibor M. Intravascular oxygen saturation in retinal vessels in normal subjects and open-angle glaucoma subjects. Acta Ophthalmol Scand. 2006;84(3):289-295.

4. Costa VP, Sergott RC, Smith M, et al. Color Doppler imaging in glaucoma patients with asymmetric optic cups. J Glaucoma. 1994; 3(Suppl 1):S91.

5. Sigal IA, Ethier CR. Biomechanics of the optic nerve head. Exp Eye Res. 2009;88(4):799-807.

6. Flammer J, Mozaffarieh M. What is the present pathogenetic concept of glaucomatous optic neuropathy? Surv Ophthalmol. 2007;52(Suppl 2): S162-S173.

7. Resch H, Garhofer G, Fuchsjäger-Mayrl G, Hommer A, Schmetterer L. Endothelial dysfunction in glaucoma. Acta Ophthalmol. 2009;87(1): 4-12.

8. Moore D, Harris A, Wudunn D, Kheradiya N, Siesky B. Dysfunctional regulation of ocular blood flow: a risk factor for glaucoma? Clin Ophthalmol. 2008;2(4):849-861.

9. Yin ZQ, Vaegan, Millar TJ, Beaumont P, Sarks S. Widespread choroidal insufficiency in primary open-angle glaucoma. J Glaucoma. 1997; 6(1):23-32.

10. Kerr J, Nelson P, O'Brien C. A comparison of ocular blood flow in untreated primary open-angle glaucoma and ocular hypertension. Am J Ophthalmol. 1998;126(1):42-51.

11. Rojanapongpun P, Drance SM, Morrison BJ. Ophthalmic artery flow velocity in glaucomatous and normal subjects. Br J Ophthalmol. 1993; 77(1):25-29.

12. Butt Z, McKillop G, O'Brien C, Allan P, Aspinall P. Measurement of ocular blood flow velocity using colour Doppler imaging in low tension glaucoma. Eye (Lond). 1995;9(Pt 1):29-33.

13. Galassi F, Sodi A, Ucci F, Harris A, Chung HS. Ocular haemodynamics in glaucoma associated with high myopia. Int Ophthalmol. 1998; 22(5):299-305.

14. Chung HS, Harris A, Evans DW, Kagemann L, Garzozi HJ, Martin B. Vascular aspects in the pathophysiology of glaucomatous optic neuropathy. Surv Ophthalmol. 1999;43(Suppl 1):S43-S50.

15. Michelson G, Langhans MJ, Harazny J, Dichtl A. Visual field defect and perfusion of the juxtapapillary retina and the neuroretinal rim area in primary open-angle glaucoma. Graefes Arch Clin Exp Ophthalmol. 1998;236(2):80-85.

16. Plange N, Kaup M, Arend O, Remky A. Asymmetric visual field loss and retrobulbar haemodynamics in primary open-angle glaucoma. Graefes Arch Clin Exp Ophthalmol. 2006;244(8):978-983.

17. Sato EA, Ohtake Y, Shinoda K, Mashima Y, Kimura I. Decreased blood flow at neuroretinal rim of optic nerve head corresponds with visual field deficit in eyes with normal tension glaucoma. Graefes Arch Clin Exp Ophthalmol. 2006;244(7):795-801.

18. Leighton DA, Phillips CI. Systemic blood pressure in open-angle glaucoma, low tension glaucoma, and the normal eye. Br JOphthalmol. 1972;56(6):447-453. 
19. Hayreh SS, Zimmerman MB, Podhajsky P, Alward WL. Nocturnal arterial hypotension and its role in optic nerve head and ocular ischemic disorders. Am J Ophthalmol. 1994;117(5):603-624.

20. Drance S, Anderson DR, Schulzer M, Group CN-TGS. Risk factors for progression of visual field abnormalities in normal-tension glaucoma. Am J Ophthalmol. 2001;131(6):699-708.

21. Harris A, Harris M, Biller J, et al. Aging affects the retrobulbar circulation differently in women and men. Arch Ophthalmol. 2000;118(8): 1076-1080.

22. Quigley HA, West SK, Rodriguez J, Munoz B, Klein R, Snyder R. The prevalence of glaucoma in a population-based study of Hispanic subjects: Proyecto VER. Arch Ophthalmol. 2001;119(12):1819-1826.

23. Bonomi L, Marchini G, Marraffa M, Bernardi P, Morbio R, Varotto A. Vascular risk factors for primary open angle glaucoma: the EgnaNeumarkt Study. Ophthalmology. 2000;107(7):1287-1293.

24. Leske MC, Wu SY, Nemesure B, Hennis A. Incident open-angle glaucoma and blood pressure. Arch Ophthalmol. 2002;120(7):954-959.

25. Grieshaber MC, Flammer J. Blood flow in glaucoma. Curr Opin Ophthalmol. 2005;16(2):79-83.

26. Vandewalle E, Abegão Pinto L, Olafsdottir OB, et al. Oximetry in glaucoma: correlation of metabolic change with structural and functional damage. Acta Ophthalmol. 2014;92(2):105-110.

27. Tezel G, Wax MB. Hypoxia-inducible factor 1alpha in the glaucomatous retina and optic nerve head. Arch Ophthalmol. 2004;122(9): $1348-1356$.

28. Stefánsson E, Pedersen DB, Jensen PK, et al. Optic nerve oxygenation. Prog Retin Eye Res. 2005;24(3):307-332.

29. Harris A, Kagemann L, Ehrlich R, Rospigliosi C, Moore D, Siesky B. Measuring and interpreting ocular blood flow and metabolism in glaucoma. Can J Ophthalmol. 2008;43(3):328-336.

30. Yap ZL, Ong C, Lee YF, et al. Retinal oximetry in subjects with glaucomatous hemifield asymmetry. J Glaucoma. 2017;26(4):367-372.

31. Kagemann L, Wollstein G, Wojtkowski M, et al. Spectral oximetry assessed with high-speed ultra-high-resolution optical coherence tomography. J Biomed Opt. 2007;12(4):041212.

32. Harris A, Dinn RB, Kagemann L, Rechtman E. A review of methods for human retinal oximetry. Ophthalmic Surg Lasers Imaging. 2003;34(2): 152-164.

33. Palsson O, Geirsdottir A, Hardarson SH, Olafsdottir OB, Kristjansdottir JV, Stefánsson E. Retinal oximetry images must be standardized: a methodological analysis. Invest Ophthalmol Vis Sci. 2012;53(4): 1729-1733.

34. Blondal R, Sturludottir MK, Hardarson SH, Halldorsson GH, Stefánsson E. Reliability of vessel diameter measurements with a retinal oximeter. Graefes Arch Clin Exp Ophthalmol. 2011;249(9):1311-1317.

35. Hardarson SH. Retinal oximetry. Acta Ophthalmol. 2013;91(5): 489-490.

36. Ramella-Roman JC, Mathews SA. Spectroscopic measurements of oxygen saturation in the retina. IEEE J Sel Top Quantum Electro. 2007(13):1697-1703.

37. Ramella-Roman JC, Mathews SA, Kandimalla H, et al. Measurement of oxygen saturation in the retina with a spectroscopic sensitive multi aperture camera. Opt Express. 2008;16(9):6170-6182.

38. Harvey AR, Fletcher-Holmes DW, Gorman A, Altenbach K, Arlt J, Read ND. Spectral imaging in a snapshot. Spectral Imaging Instrum Appl Anal III. 2005(5694):110-119.

Clinical Ophthalmology

\section{Publish your work in this journal}

Clinical Ophthalmology is an international, peer-reviewed journal covering all subspecialties within ophthalmology. Key topics include: Optometry; Visual science; Pharmacology and drug therapy in eye diseases; Basic Sciences; Primary and Secondary eye care; Patient Safety and Quality of Care Improvements. This journal is indexed on Submit your manuscript here: http://www.dovepress.com/clinical-ophthalmology-journal
39. Johnson WR, Wilson DW, Fink W, Humayun M, Bearman G. Snapshot hyperspectral imaging in ophthalmology. J Biomed Opt. 2007(12): 014036.

40. Kashani AH, Kirkman E, Martin G, Humayun MS. Hyperspectral computed tomographic imaging spectroscopy of vascular oxygen gradients in the rabbit retina in vivo. PLoS One. 2011;6(9):e24482.

41. Khoobehi B, Beach JM, Kawano H. Hyperspectral imaging for measurement of oxygen saturation in the optic nerve head. Invest Ophthalmol Vis Sci. 2004;45(5):1464-1472.

42. Beach J, Ning J, Khoobehi B. Oxygen saturation in optic nerve head structures by hyperspectral image analysis. Curr Eye Res. 2007;32(2): 161-170.

43. Yoneya S, Saito T, Nishiyama Y, et al. Retinal oxygen saturation levels in patients with central retinal vein occlusion. Ophthalmology. 2002;109(8):1521-1526.

44. Desjardins M, Sylvestre JP, Jafari R, et al. Preliminary investigation of multispectral retinal tissue oximetry mapping using a hyperspectral retinal camera. Exp Eye Res. 2016;146:330-340.

45. Gonzalez-Hernandez M, Sigut Saavedra J, Gonzalez de la Rosa M. Relationship between retinal nerve fiber layer thickness and hemoglobin present in the optic nerve head in glaucoma. $J$ Ophthalmol. 2017;2017:2340236.

46. Mordant DJ, Al-Abboud I, Muyo G, Gorman A, Harvey AR, McNaught AI. Oxygen saturation measurements of the retinal vasculature in treated asymmetrical primary open-angle glaucoma using hyperspectral imaging. Eye (Lond). 2014;28(10):1190-1200.

47. Hardarson SH, Gottfredsdottir MS, Halldorsson GH, et al. Glaucoma filtration surgery and retinal oxygen saturation. Invest Ophthalmol Vis Sci. 2009;50(11):5247-5250.

48. Ito M, Murayama K, Deguchi T, et al. Oxygen saturation levels in the juxta-papillary retina in eyes with glaucoma. Exp Eye Res. 2008; 86(3):512-518.

49. Cheng CS, Lee YF, Ong C, et al. Inter-eye comparison of retinal oximetry and vessel caliber between eyes with asymmetrical glaucoma severity in different glaucoma subtypes. Clin Ophthalmol. 2016;10: $1315-1321$.

50. Siesky B, Harris A, Ehrlich R, et al. Short-term effects of brimonidine/ timolol and dorzolamide/timolol on ocular perfusion pressure and blood flow in glaucoma. Adv Ther. 2012;29(1):53-63.

51. Traustason S, Hardarson SH, Gottfredsdottir MS, et al. Dorzolamidetimolol combination and retinal vessel oxygen saturation in patients with glaucoma or ocular hypertension. Br J Ophthalmol. 2009;93(8): 1064-1067.

52. Siesky B, Harris A, Cantor LB, et al. A comparative study of the effects of brinzolamide and dorzolamide on retinal oxygen saturation and ocular microcirculation in patients with primary open-angle glaucoma. $\mathrm{Br} \mathrm{J}$ Ophthalmol. 2008;92(4):500-504.

53. Nitta E, Hirooka K, Shimazaki T, et al. Retinal oxygen saturation before and after glaucoma surgery. Acta Ophthalmol. 2016;95(5): e350-e353.

54. Hardarson SH, Stefánsson E. Retinal oxygen saturation is altered in diabetic retinopathy. Br J Ophthalmol. 2012;96(4):560-563.

55. Imedos Systems UG, the Pioneer of retinal Vessel Analysis. http:// www.imedos.de/Oxygen-Module.147.0.html

\section{Dovepress}

PubMed Central and CAS, and is the official journal of The Society of Clinical Ophthalmology (SCO). The manuscript management system is completely online and includes a very quick and fair peer-review system, which is all easy to use. Visit http://www.dovepress.com/ testimonials.php to read real quotes from published authors. 